\title{
Characterization of laser beams: theory and application in laser-assisted bonding process
}

\author{
Wagno Alves Braganca Jr. ${ }^{*} *$ and KyungOe Kim \\ JCET Group Co. Ltd., Incheon, Republic of Korea
}

\begin{abstract}
The International Organization for Standardization (ISO), in collaboration with the academic community, has developed and published a comprehensive system of standards for the characterization of optical components and laser beams. Our study offers a pedagogical introduction to the theory and application of laser beam characterization in accordance with ISO 13694:2018 and ISO 11145:2018. The characterizing parameters of each ISO standard are presented, defined mathematically, and explained in detail through the use of a minimum working example. A laser beam applied to an actual industrial process [laser-assisted bonding (LAB) process in the semiconductor packaging industry] is thoroughly analyzed to help the reader understand and interpret the characterizing results. Background noise correction and unit conversion methods are also discussed. We target researchers new to the field of laser beam analysis who want the acquire background knowledge, as well as more experienced researchers who want to implement their own laser beam analysis software and propose new definitions for their specific application. Although our study deals with only two ISO standards, the methods covered in our study can be easily applied to other norms that fit the reader's application. Our study should not be treated as a full review of the LAB process nor of the beam profiling instruments and measurement principles. These are only mentioned to enhance the reader's learning experience. (C) 2021 Society of Photo-Optical Instrumentation Engineers (SPIE) [DOI: 10.1117/1.OE.60.6 .060801]
\end{abstract}

Keywords: beam characterization; beam profiling; International Organization for Standardization; theory; application; laser-assisted bonding.

Paper 20210294T received Mar. 17, 2021; accepted for publication Jun. 2, 2021; published online Jun. 17, 2021.

\section{Introduction}

The International Organization for Standardization (ISO), in collaboration with the International Electrotechnical Commission and the EUREKA-project EUROLASER: characterization of optical components and laser beams (CHOCLAB), has developed and published a comprehensive system of standards over the past 30 years. ${ }^{1}$ The academic community has been actively engaged in this work as well, contributing a wide range of publications that cover the motivation and process behind the establishment of laser-related norms, ${ }^{2}$ the development of measuring principles and instruments, ${ }^{3}$ the debate over the definition of characterizing parameters, ${ }^{4,5}$ and the discussion over test procedures. ${ }^{6}$ Practical characterization of laser beams has also been reported, ${ }^{7-10}$ and improvements to the ISO standards have been proposed. ${ }^{11,12}$ Furthermore, computer algorithms and data processing for the visualization and characterization of laser beams have been investigated in an effort to reduce the user's dependency on third-party, proprietary software. $^{13,14}$

However, none of the available publications explain the characterizing parameters of laser beams in a didactic manner suited to newcomers to the field. This paper offers a pedagogical introduction to the theory of laser beam characterization through the use of a minimum working example (MWE) that illustrates how each ISO definition is computed. In addition, this paper presents the characterization of a laser beam applied to an actual industrial process [laser-assisted

*Address all correspondence to Wagno Alves Braganca, wagnojunior.ab@jcetglobal.com 
bonding (LAB) process in the semiconductor packaging industry], which will help the reader understand and interpret the characterization results.

We target researchers new to the field of laser beam analysis who want to acquire background knowledge, as well as more experienced researches who want to implement their own laser beam analysis software and propose new definitions for their specific application. This paper should not be treated as a full review of LAB process, nor should it be considered as a summary of the instruments and measurement principles related to laser beam profiling. These are solely mentioned to give the reader a basic understanding of how the laser beam data are collected and stored and also to present the reader a concrete example that will enhance their comprehension.

\section{Process Overview}

\subsection{Laser-Assisted Bonding}

$\mathrm{LAB}$ is a semiconductor packaging process in which a laser beam is selectively applied to a target flip-chip die and/or surface-mount component for less than a couple of seconds. ${ }^{15-20}$ The interaction between the laser beam and the target device increases its temperature, thus allowing it to be metallurgically bonded to a substrate, interposer, or another die. The LAB system (Fig. 1) is composed of a high-power diode laser source, an optical fiber, a collimator, and a homogenizer that produces a flat-top laser beam. The reader is encouraged to refer to Refs. 21-23 for a comprehensive study on the theory and techniques of laser beam shaping, including how flat-top laser beams are formed. The laser source operates in the continuous-wave mode, in the near-infrared wavelength of $980 \mathrm{~nm}$, and with a maximum power of $2 \mathrm{~kW}$. The beam size is 9 to $45 \mathrm{~mm}$ and the focal distance is $240 \mathrm{~mm}$.

The power density distribution and spatial properties of the laser beam are crucial for the overall robustness of the LAB process (Fig. 2). Open-circuit issues may occur at the solder bumps if some areas of the target device do not receive sufficient laser energy. Similarly, short-circuit issues may occur at the solder bumps if some areas of the target device receive excessive laser energy. In extreme cases, the target device might even burn out, damaging the electronic component and the LAB tooling. Therefore, it is absolutely essential that all areas of the target device receive the same amount of laser energy, thus ensuring a uniform temperature distribution and a reliable solder joint.

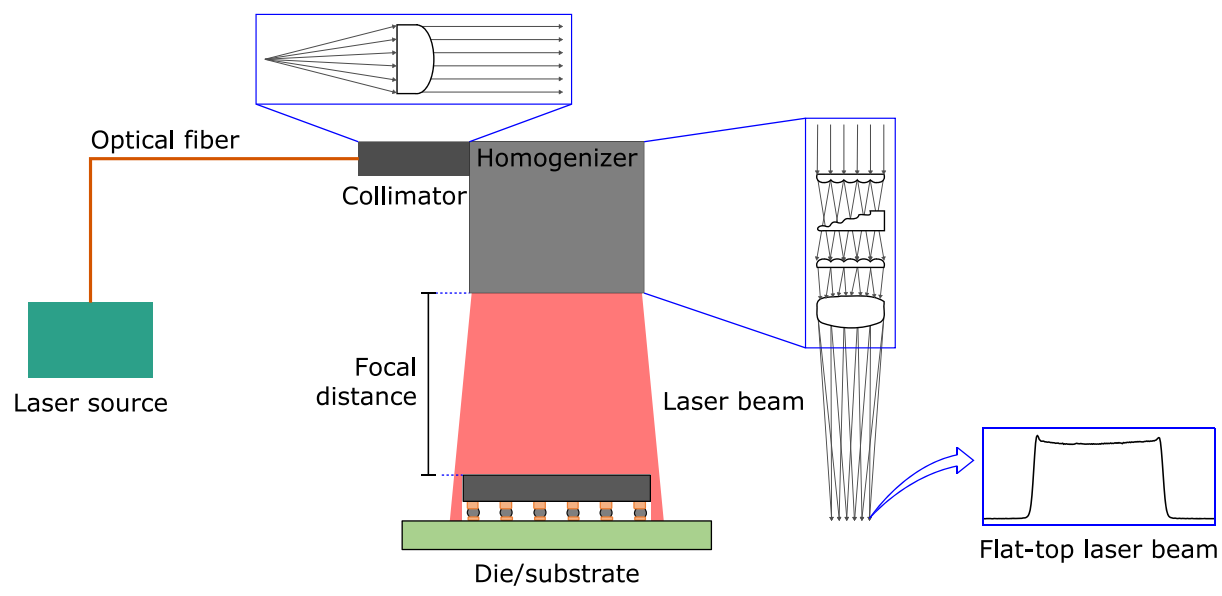

Fig. 1 LAB overview. The diode laser source generates a continuous wave, 2 kW, 980-nmwavelength laser beam; the optical fiber connects the laser source to a collimator; and the beam homogenizer produces a flat-top laser beam. The beam size is 9 to $45 \mathrm{~mm}$ and the focal distance is $240 \mathrm{~mm}$. 


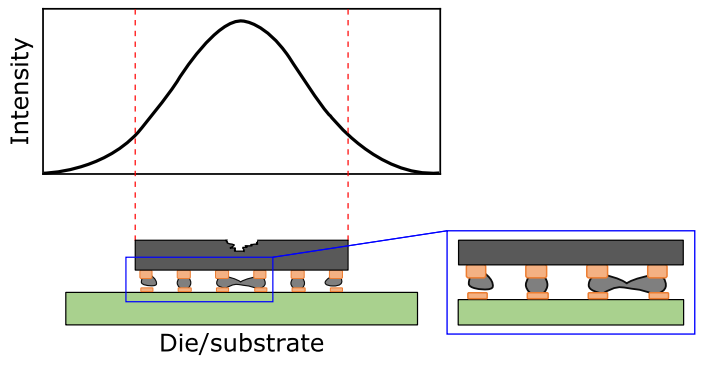

Fig. 2 Device-level issues related to the power density distribution in the LAB process. Opencircuit issues are related to insufficient laser power, whereas short-circuit issues are related to excessive laser power. In extreme cases, the target device might even burn out. The inset image shows the magnified view of a bump with open-circuit issue, with no issue, and with a short-circuit issue.

\subsection{Measurement Setup}

The beam profiling measurement tool used in this study is a BeamMonitor BM+ 100 from PRIMES $^{24}$ (Fig. 3). It features a DFY-PS photodiode sensor with automatic sensitivity adaptation in the 400-to 1100 -nm wavelength range. The aperture size of $100 \mathrm{~mm}$ supports a maximum measuring window size of $100 \mathrm{~mm}$ and a maximum beam size of $70 \mathrm{~mm}$, which covers the entire dimension range of the LAB system and excludes the need of extra optics for beam size reduction. The maximum power density of $10 \mathrm{~kW} / \mathrm{cm}^{2}$ supports the maximum power density of the LAB system and excludes the need of extra power attenuation. The BeamMonitor BM+100 is used in association with a water-cooled laser beam dump.

The laser beam is scanned by a rotating measuring tip that moves along the length of the laser beam dump. A mirror mounted on the measuring tip deflects a fraction of the light into the laser detector circuitry, which digitizes the electrical signal with a 16-bit analog-digital converter. The number of measured lines along the length of the laser beam dump determines the pixel resolution in the $y$ axis, and the number of scanning points per line determines the pixel resolution in the $x$ axis. The maximum supported pixel resolution is $1024 \mathrm{px} \times 1024 \mathrm{px}$, but $128 \mathrm{px} \times 128 \mathrm{px}$ is generally sufficient to yield high-precision analysis. The power density (measured in analog-digital counts per pixel-ADC/px) at a specific $x-y$ position is recorded in a corresponding pixel, and the collection of pixels gives the power density distribution of the irradiating laser beam (Fig. 4).

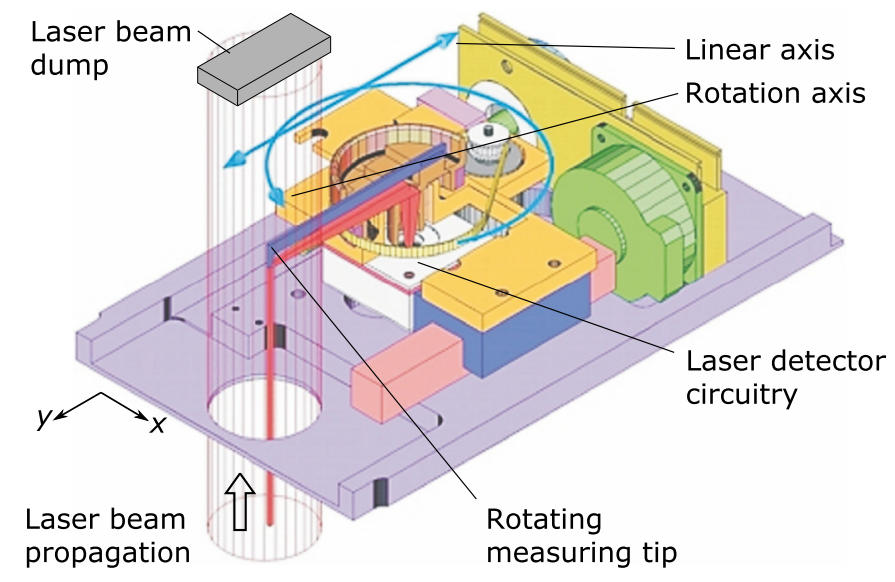

Fig. 3 BeamMonitor BM+ 100 from PRIMES. A rotating measuring tip deflects a fraction of the laser beam into the laser detector circuitry. Image courtesy of PRIMES GmbH. 


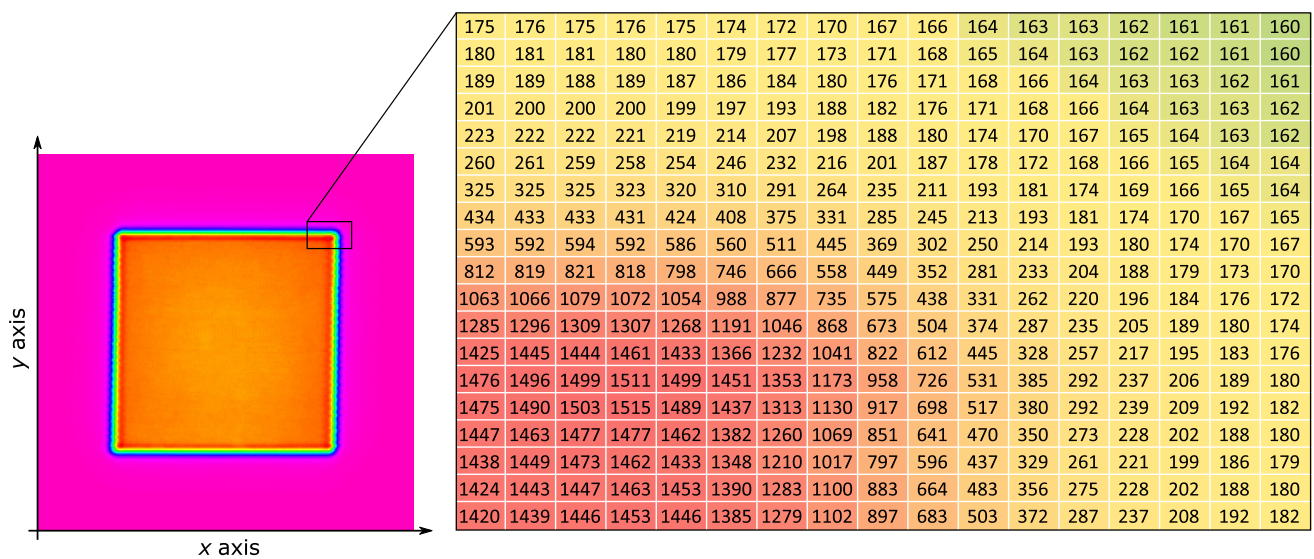

Fig. 4 Power density distribution of a flat-top laser beam in the LAB process. The inset image shows the magnified view of the top-right corner of the power density distribution. The power density at a specific pixel is measured in $A D C / p x$.

\section{ISO Standards}

ISO $13694: 2018^{25}$ and ISO $11145: 2018^{26}$ are the two most pertinent standards related to the LAB process; therefore, only these two norms will be discussed in this paper. However, the methods learned from this paper can be easily applied to other norms that fit the reader's application. An MWE (Fig. 5) is used to illustrate how each ISO definition is computed. The reader is encouraged to follow the calculations step-by-step by hand, spread-sheet software, or generalpurpose programming language. The following definitions were implemented using the Python programming language, and the source file and raw data can be made available upon reasonable request to the corresponding author. The reader is encouraged to refer to Ref. 25 for more information on measurement arrangement and test equipment and procedures, as these topics are not the focus of this paper.

\subsection{Characterizing Parameters}

\subsubsection{Power density distribution}

Power density distribution ${ }^{25} E(x, y, z)$ is the set of all power densities at location $z$ for all coordinates $(x, y)$. The power density distribution of the MWE (Fig. 5) is the collection of pixels from 0 to 6 in both $x$ and $y$ axes.

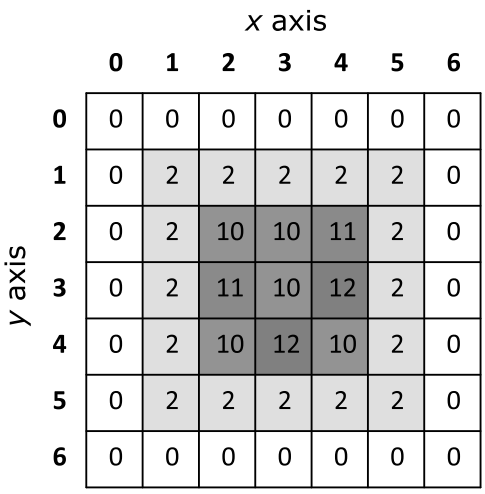

Fig. 5 The MWE consists of a simple, fictional power density distribution with the purpose of illustrating the definitions of ISO 13694:2018 and ISO 11145:2018. It has a 7 px $\times 7$ px resolution and the values of each pixel are analogous to those of Fig. 4. 


\subsubsection{Power density}

Power density ${ }^{25} E\left(x_{p}, y_{p}, z\right)$ is the power density at location $z$ for coordinate $\left(x_{p}, y_{p}\right)$. The power density of the MWE (Fig. 5) is $E(2,4, z)=10$.

\subsubsection{Maximum power density}

Maximum power density ${ }^{25} E_{\max }(z)$ is the maximum of the power density distribution $E(x, y, z)$ at location $z$ for coordinate $\left(x_{\max }, y_{\max }, z\right)$. The maximum power density of the MWE (Fig. 5) is $E_{\max }(z)=12$ for coordinates $(3,4)$ and $(4,3)$.

\subsubsection{Power}

Power $^{25} P(z)$ is the sum of the power density $E\left(x_{p}, y_{p}, z\right)$ at location $z$ evaluated over all coordinates $(x, y)$ of the power density distribution $E(x, y, z) . P(z)$ is mathematically defined as

$$
P(z)=\sum_{x} \sum_{y} E(x, y, z) .
$$

The power of the MWE (Fig. 5) is $P(z)=128$.

\subsubsection{Clip-level power density}

Clip-level power density ${ }^{25} E_{\eta \mathrm{CL}}(z)$ is the fraction $\eta$ of the maximum power density $E_{\max }(z)$ at location $z . E_{\eta \mathrm{CL}}(z)$ is mathematically defined as

$$
E_{\eta \mathrm{CL}}(z)=\eta E_{\max }(z)
$$

where $0 \leq \eta<1$. The clip-level power density of the MWE (Fig. 5) is $E_{\eta \mathrm{CL}}=9.60$ considering a clip-level of $\eta=0.80$.

\subsubsection{Clip-level power}

Clip-level power ${ }^{25} P_{\eta}(z)$ is the sum of the power density $E\left(x_{p}, y_{p}, z\right)$ at location $z$ evaluated only over locations $(x, y)$ for which $E(x, y, z)>E_{\eta \mathrm{CL}}(z) . P_{\eta}(z)$ is mathematically expressed as

$$
P_{\eta}(z)=\sum_{x} \sum_{y}\left[E(x, y, z)>E_{\eta \mathrm{CL}}(z)\right] .
$$

The clip-level power of the MWE (Fig. 5) is $P_{\eta}(z)=96$ considering a clip-level of $\eta=0.80$.

\subsubsection{Fractional power}

Fractional power ${ }^{25} f_{\eta}(z)$ is the fraction of the clip-level power $P_{\eta}(z)$ for a given $\eta$ to the total power $P(z)$ in the distribution at location $z \cdot f_{\eta}(z)$ is mathematically expressed as

$$
f_{\eta}(z)=\frac{P_{\eta}(z)}{P(z)}
$$

where $0 \leq f_{\eta}(z) \leq 1$. The fractional power of the MWE (Fig. 5) is $f_{\eta}(z)=0.75$ considering a clip level of $\eta=0.80$, which means that $75 \%$ of the total power has a value $>9.60$.

\subsubsection{Beam centroid}

Beam centroid $^{26}(\bar{x}(z), \bar{y}(z))$ is the coordinate of the first-order moment of the power density distribution $E(x, y, z)$ at location $z \cdot(\bar{x}(z), \bar{y}(z))$ is mathematically defined as 


$$
\begin{aligned}
& \bar{x}(z)=\frac{\sum_{x} \sum_{y} x \cdot E(x, y, z)}{\sum_{x} \sum_{y} E(x, y, z)}, \\
& \bar{y}(z)=\frac{\sum_{x} \sum_{y} y \cdot E(x, y, z)}{\sum_{x} \sum_{y} E(x, y, z)},
\end{aligned}
$$

where the nominators and denominators are the first-order moment and the zeroth-order moment of the distribution, respectively.

The first-order moment (Fig. 6) computes the value of each pixel weighted by its corresponding column index (if the moment is calculated about the $x$ axis) and by its corresponding row index (if the moment is calculated about the $y$ axis). For instance, the first-order moment about the $x$ axis is calculated by multiplying the values of each pixel in the first column (index 0) by 0 , in the second column (index 1) by 1, in the third column (index 2) by 2, and so on [Fig. 6(a)]. Similarly, the first-order moment about the $y$ axis is calculated by multiplying the values of each pixel in the first row (index 0 ) by 0 , in the second row (index 1 ) by 1 , in the third row (index 2 ) by 2 , and so on [Fig. 6(b)]. The values of each pixel are subsequently added. The beam centroid of the MWE (Fig. 5) is $\bar{x}(z)=3$ and $\bar{y}(z)=3$.

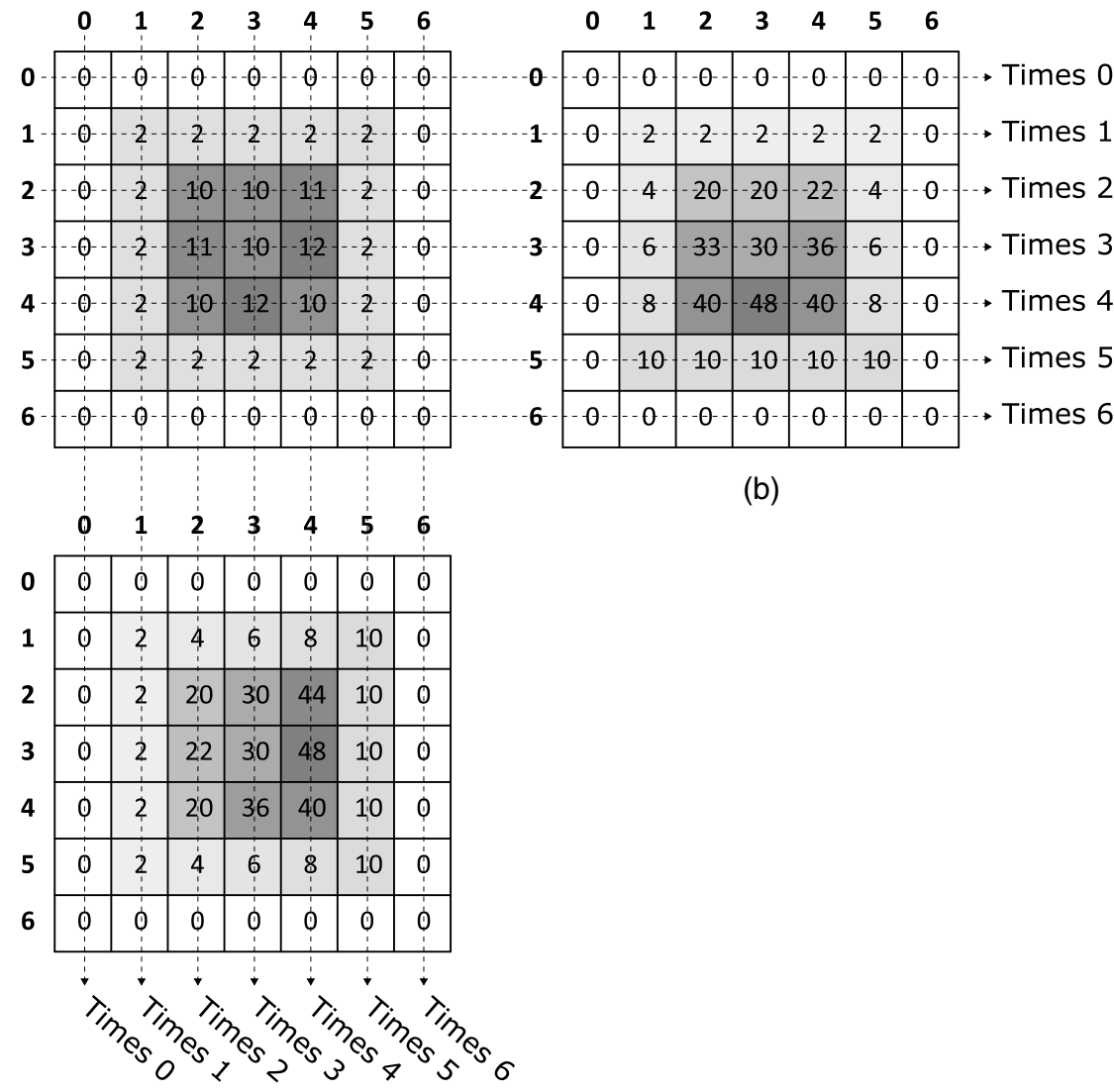

(a)

Fig. 6 Visual explanation of the first-order moment. (a) The first-order moment about the $x$ axis is computed by multiplying the values of each pixel in the original power density distribution by its corresponding column index. (b) The first-order moment about the $y$ axis is calculated by multiplying the values of each pixel in the original power density distribution by its corresponding row index. 


\subsubsection{Beam positional stability}

Beam positional stability ${ }^{26}\left(\Delta_{x}(z), \Delta_{y}(z)\right)$ is four times the standard deviation of the measured beam positional movement at location $z \cdot\left(\Delta_{x}(z), \Delta_{y}(z)\right)$ is expressed mathematically as

$$
\begin{aligned}
& \Delta_{x}(z)=4 \sqrt{\frac{\sum_{i=1}^{N}\left[\bar{x}(z)_{i}-\overline{\bar{x}(z)}\right]^{2}}{N-1}}, \\
& \Delta_{y}(z)=4 \sqrt{\frac{\sum_{i=1}^{N}\left[\bar{y}(z)_{i}-\overline{\bar{y}(z)}\right]^{2}}{N-1}},
\end{aligned}
$$

where $(\bar{x}(z), \bar{y}(z))_{i}$ is the $i$ 'th beam centroid at location $z,(\overline{\bar{x}(z)}, \overline{\bar{y}}(z))$ is the mean beam centroid at location $z$, and $N$ is the number of measurements. Since the MWE (Fig. 5) consists of a single measurement, it is not possible to calculate its beam positional stability.

\subsubsection{Beam width}

Beam width ${ }^{26}\left(d_{\sigma x}(z), d_{\sigma y}(z)\right)$ is four times the square root of the second-order moment of the power density distribution $E(x, y, z)$ about the beam centroid $(\bar{x}(z), \bar{y}(z))$ at location $z$. $\left(d_{\sigma x}(z), d_{\sigma y}(z)\right)$ is mathematically expressed as

$$
\begin{aligned}
& d_{\sigma x}(z)=4 \sqrt{\frac{\sum_{x} \sum_{y}[x-\bar{x}(z)]^{2} \cdot E(x, y, z)}{\sum_{x} \sum_{y} E(x, y, z)}}, \\
& d_{\sigma y}(z)=4 \sqrt{\frac{\sum_{x} \sum_{y}[y-\bar{y}(z)]^{2} \cdot E(x, y, z)}{\sum_{x} \sum_{y} E(x, y, z)}},
\end{aligned}
$$

where the nominators and denominators are the second-order moment and the zeroth-order moment of the distribution, respectively.

The second-order moment (Fig. 7) computes the value of each pixel weighted by the square of the distance from its corresponding column index to the beam $x$ centroid (if the moment is calculated about the $x$ axis) and by the square of the distance from its corresponding row index to the beam $y$ centroid (if the moment is calculated about the $y$ axis). For example, the second-order moment about the $x$ axis is calculated by multiplying the values of each pixel in the first column (index 0 ) by $(0-3)^{2}=9$, in the second column (index 1) by $(1-3)^{2}=4$, in the third column (index 2) by $(2-3)^{2}=1$, and so on [Fig. 7(a)]. Likewise, the second-order moment about the $y$ axis is calculated by multiplying the values of each pixel in the first row (index 0) by $(0-3)^{2}=9$, in the second row (index 1) by $(1-3)^{2}=4$, in the third row (index 2) by $(2-3)^{2}=1$, and so forth [Fig. 7(b)]. The values of each pixel are subsequently added. The bean width of the MWE (Fig. 5) is $d_{\sigma x}(z)=4.36$ and $d_{\sigma y}(z)=4.34$.

\subsubsection{Beam aspect ratio}

Beam aspect ratio $^{26} \varepsilon(z)$ quantifies the squareness of a power density distribution $E(x, y, z)$ at location $z . \varepsilon(z)$ is mathematically defined as

$$
\varepsilon(z)=\frac{d_{\sigma y}(z)}{d_{\sigma x}(z)} .
$$

The beam aspect ratio of the MWE (Fig. 5) is $\varepsilon(z)=0.99$, which suggests a fairly square shape. 


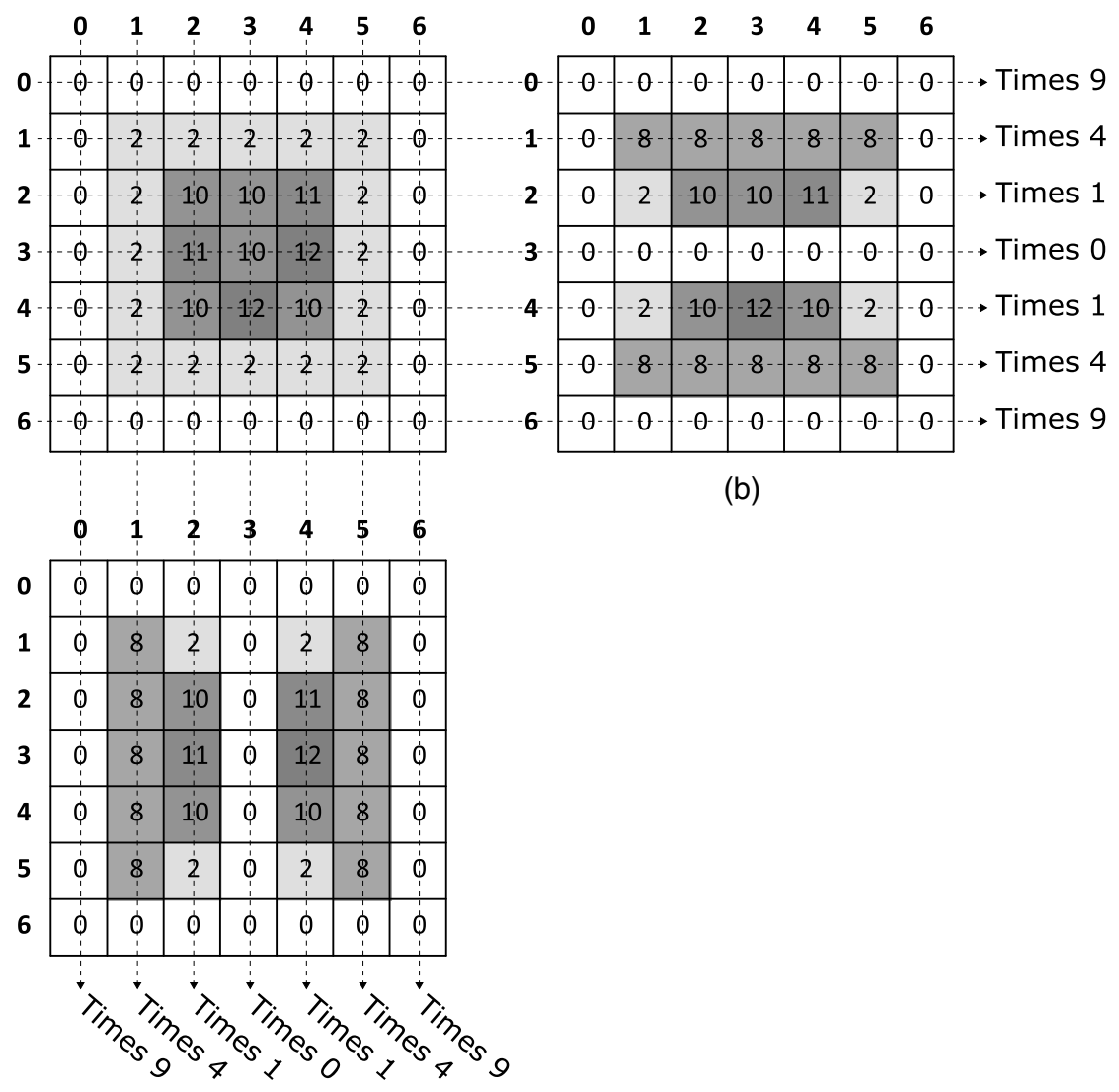

(a)

Fig. 7 Visual explanation of the second-order moment. (a) The second-order moment about the $x$ axis is computed by multiplying the values of each pixel in the original power density distribution by the square of the distance from its corresponding column index to the beam $x$ centroid. (b) The second-order moment about the $y$ axis is calculated by multiplying the values of each pixel in the original power density distribution by the square of the distance from its corresponding row index to the beam $y$ centroid.

\subsubsection{Clip-level irradiation area}

Clip-level irradiation area ${ }^{25} A_{\eta}^{i}(z)$ is the irradiation area at location $z$, for which the power density $E\left(x_{p}, y_{p}, z\right)$ exceeds the clip-level power density $E_{\eta \mathrm{CL}}(z) . A_{\eta}^{i}(z)$ is mathematically expressed as

$$
A_{\eta}^{i}(z)=\operatorname{COUNT}\left[E(x, y, z)>E_{\eta \mathrm{CL}}(z)\right]
$$

where COUNT is a function that counts how many pixels have a value greater than the clip-level power density $E_{\eta \mathrm{CL}}(z)$. The clip-level irradiation area of the MWE (Fig. 5) is $A_{\eta}^{i}(z)=9$ considering a clip-level of $\eta=0.80$.

\subsubsection{Clip-level average power density}

Clip-level average power density ${ }^{25} E_{\eta \text { ave }}(z)$ is the spatially averaged power density of the distribution at location $z . E_{\eta \text { ave }}(z)$ is mathematically defined as

$$
E_{\eta \text { ave }}(z)=\frac{P_{\eta}(z)}{A_{\eta}^{i}(z)}
$$

The clip-level average power density of the MWE (Fig. 5) is $E_{\eta \text { ave }}(z)=10.67$ considering a clip-level of $\eta=0.80$. 


\subsubsection{Flatness factor}

Flatness factor ${ }^{25} F_{\eta}(z)$ is the ratio of the clip-level average power density $E_{\eta \text { ave }}(z)$ to the maximum power density of the distribution $E_{\max }(z)$ at location $z \cdot F_{\eta}(z)$ is mathematically defined as

$$
F_{\eta}(z)=\frac{E_{\eta \mathrm{ave}}(z)}{E_{\max }(z)}
$$

where $0<F_{\eta}(z) \leq 1$ and $F_{\eta}(z)=1$ for a perfectly flat-top power density distribution. The flatness factor of the MWE (Fig. 5) is $F_{\eta}(z)=0.89$ considering a clip-level of $\eta=0.80$, which suggests a flat distribution.

\subsubsection{Beam uniformity}

Beam uniformity ${ }^{25} U_{\eta}(z)$ is the normalized root mean square deviation of the power density distribution $E(x, y, z)$ from its clip-level average power density $E_{\eta \text { ave }}(z)$ at location $z . U_{\eta}(z)$ is mathematically expressed as

$$
U_{\eta}(z)=\frac{1}{E_{\eta \text { ave }}(z)} \sqrt{\frac{1}{A_{\eta}^{i}(z)} \sum_{x} \sum_{y}\left[E(x, y, z)-E_{\eta \mathrm{ave}}(z)\right]^{2}},
$$

where $U_{\eta}(z)=0$ indicates a completely uniform distribution having a profile with a flat-top and vertical edges. The expression $\left[E(x, y, z)-E_{\eta \text { ave }}(z)\right]^{2}$ is computed pixel by pixel (Fig. 8) and the double sum is performed only on pixels whose original value is greater than the clip-level power density $E_{\eta \mathrm{CL}}(z)$. The beam uniformity of the MWE (Fig. 5) is $U_{\eta}(z)=0.076$ considering a cliplevel of $\eta=0.80$, which indicates a uniformity of $7.6 \% \mathrm{rms}$ from its mean value at location $z$.

\subsubsection{Plateau uniformity}

Plateau uniformity ${ }^{25} U_{p}(z)$ is the quantitative measure of the homogeneity of nearly flat-top profiles. $U_{p}(z)$ is mathematically expressed as

$$
U_{p}(z)=\frac{\Delta E_{\mathrm{FWHW}}(z)}{E_{\max }(z)},
$$

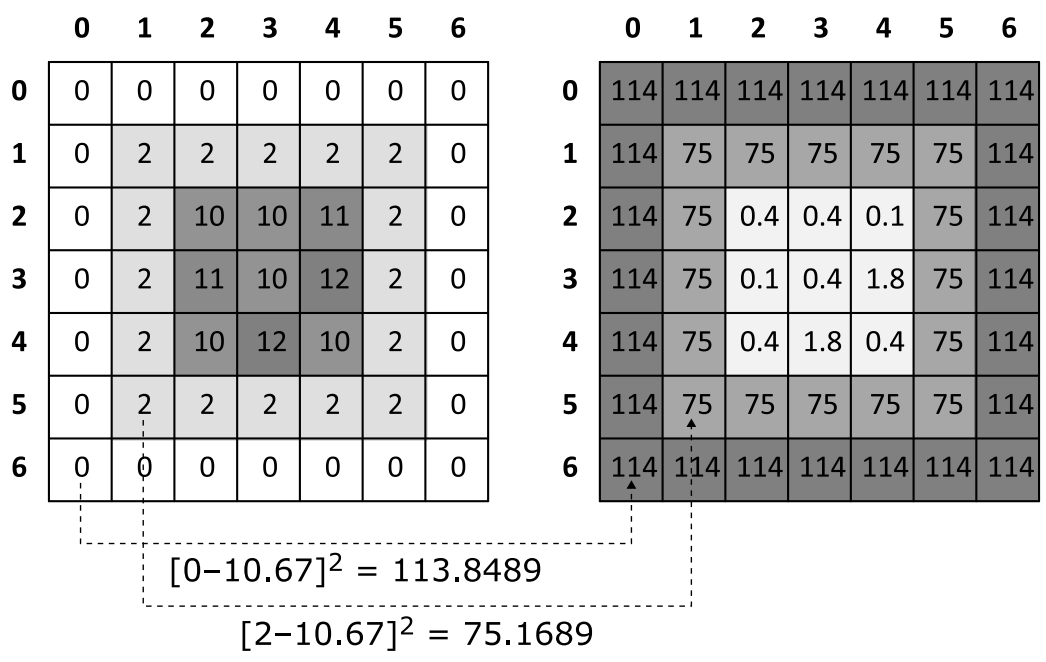

Fig. 8 Visual explanation of one of the steps to calculate the beam uniformity. The expression $\left[E(x, y, z)-E_{\eta \text { ave }}(z)\right]^{2}$ for pixel $(0,6)$ is $[0-10.67]^{2}=144$, approximately. Similarly for pixel $(1,5)$, the expression is approximately $[2-10.67]^{2}=75$. The double sum yields 6.00 . 

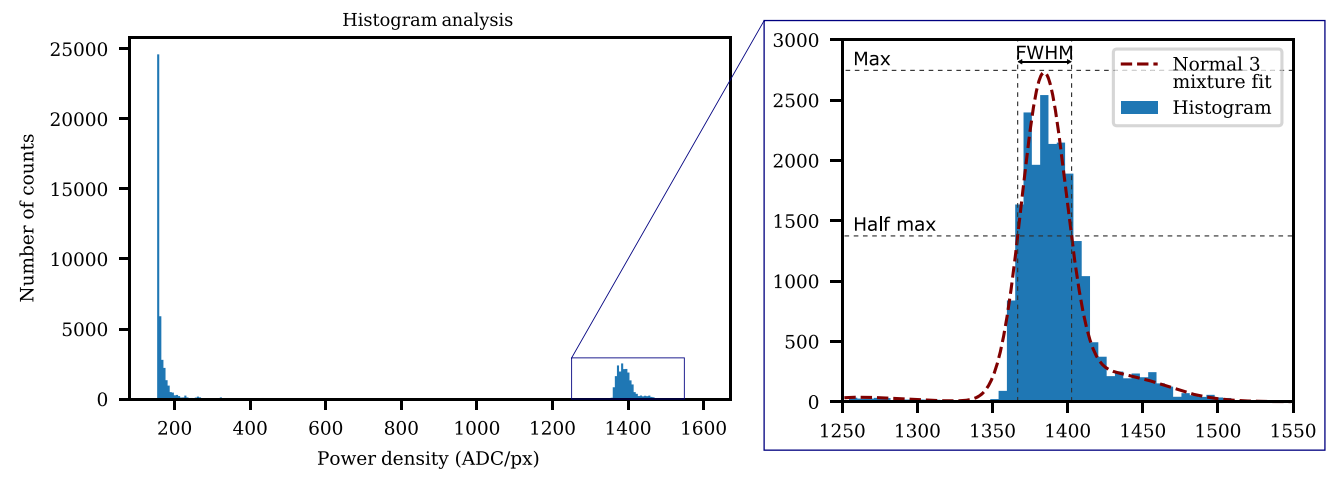

Fig. 9 Histogram of the power density distribution in Fig. 4. The histogram is divided into 256 bins since the power density distribution in Fig. 4 has a total of 65,536 data points. The inset image shows the detailed view of the histogram peak near $E_{\max }(z)$, the normal 3 mixture fit, and a visual explanation of the FWHM.

where $0<U_{p}(z)<1, \Delta E_{\mathrm{FWHM}}(z)$ is the full-width at half-maximum (FWHM) of the peak near $E_{\max }$ of the power density histogram and $U_{p}(z)=0$ for a perfectly flat-top distribution. The power density histogram (Fig. 9) typically shows two peaks: one near the background noise and digitizer baseline (low end of the $x$ axis) and another near the $E_{\max }(z)$ value (high end of the $x$ axis). If the power density histogram at around the high end of the $x$ axis is normally distributed, then a normal fit can be used to determine the FWHM. Otherwise, a normal mixture fit can be used instead (inset in Fig. 9). The histogram of the power density distribution is divided into $k$ bins, where $k$ is commonly defined as the square root of the total number of data points. The plateau uniformity of the MWE (Fig. 5) is approximately $U_{p}(z)=0.16$ considering a cliplevel of $\eta=0.80$, which indicates a homogeneous distribution.

\subsubsection{Edge steepness}

Edge steepness ${ }^{25} S_{\varepsilon, \eta}(z)$ is the normalized difference between the clip-level irradiation areas $A_{\varepsilon}^{i}(z)$ and $A_{\eta}^{i}(z) . S_{\varepsilon, \eta}(z)$ is mathematically defined as

$$
S_{\varepsilon, \eta}(z)=\frac{A_{\varepsilon}^{i}(z)-A_{\eta}^{i}(z)}{A_{\varepsilon}^{i}(z)},
$$

where $0<S_{\varepsilon, \eta}(z)<1 ; 0 \leq \varepsilon<\eta<1$; and $S_{\varepsilon, \eta}(z)=0$ for a power density distribution with perfectly vertical edges. The edge steepness of the MWE (Fig. 5) is $S_{\varepsilon, \eta}(z)=0.64$ considering a clip-level of $\varepsilon=0.10$ and $\eta=0.80$, which suggests a distribution with slight vertical edges.

\subsection{Noise Correction}

Background noise and digitizer baseline are known to negatively affect the soundness of the beam analysis, in special calculations that involve weighted integral sums, such as power and first- and second-order moment. ${ }^{7,8}$ Therefore, a noise correction method must be applied before carrying on the laser beam analysis. ISO 13649:2018 ${ }^{25}$ defines two background noise correction methods based on the dark-field of the measurement tool, that is, the power density distribution when the laser shutter is closed. The first method, background map subtraction, consists of recording the dark-field map of the detector and subtracting it pixel by pixel from the power density distribution. The second method, average background map subtraction, consists of recording the dark-field map of the detector and subtracting the average value from the power density distribution.

Apte and Gower ${ }^{7}$ introduced a non-ISO method, which consists of analyzing the histogram of the power density distribution and subtracting the most frequently occurring value from the 
power density distribution (153 ADC/px in Fig. 9). In this case, the fill factor-the ratio of the beam size to the measuring window size - should be between 0.35 and 0.70 for easy identification of the background count. The BeamMonitor BM+ 100 from PRIMES employs this method.

\section{Results and Discussion}

The size of the measuring window and the pixel resolution used in this study are correspondingly $35.1 \mathrm{~mm} \times 35.1 \mathrm{~mm}$ and $256 \mathrm{px} \times 256 \mathrm{px}$. A 700-W, flat-top laser beam was impinged onto the beam profiling measurement tool, and the power density distribution was recorded 10 consecutive times (Fig. 10). The noise correction method based on the histogram analysis was applied to each of the 10 measurements, as described in Sec. 3.2. The characterizing parameters discussed in Sec. 3.1 were analyzed using the Python programming language. The results considering $\varepsilon=0.10$ and $\eta=0.80$ are summarized in Table 1 according to their category.

\subsection{Unit Conversion}

\subsubsection{Measurement unit of power}

The measurement unit of power can be converted from ADC to $W$ by correlating the calculated power $P(z)(28,576,928.9160$ ADC) with the output laser power. Since the entire optical system has a loss of $\sim 5 \%$, the output laser power should be verified by a calibrated power meter. The power meter used in this study is a PowerMax-Pro HP from Coherent. It features a thin-film detector with a calibration uncertainty of $\pm 2 \%$ at $810 \mathrm{~nm}$, a spectral compensation accuracy of $\pm 5 \%$, a power linearity of $\pm 2 \%$, and a spatial uniformity of $\pm 5 \%$.

The output laser power was measured three consecutive times, and the average value was confirmed as $700 \mathrm{~W}$. Therefore, the conversion factor of power is $\frac{700 \mathrm{~W}}{28.576 .928 .9160 \mathrm{ADC}}=$ $2.4495 \times 10^{-5} \frac{\mathrm{W}}{\mathrm{ADC}}$.

\subsubsection{Measurement unit of length}

The measurement unit of length can be converted from $\mathrm{px}$ to $\mathrm{mm}$ by correlating the pixel resolution $(256 \mathrm{px} \times 256 \mathrm{px})$ with the size of the measuring window $(35.1 \mathrm{~mm} \times 35.1 \mathrm{~mm})$. The conversion factor of length is $\frac{35.1 \mathrm{~mm}}{256 \mathrm{px}}=0.1371 \frac{\mathrm{mm}}{\mathrm{px}}$.

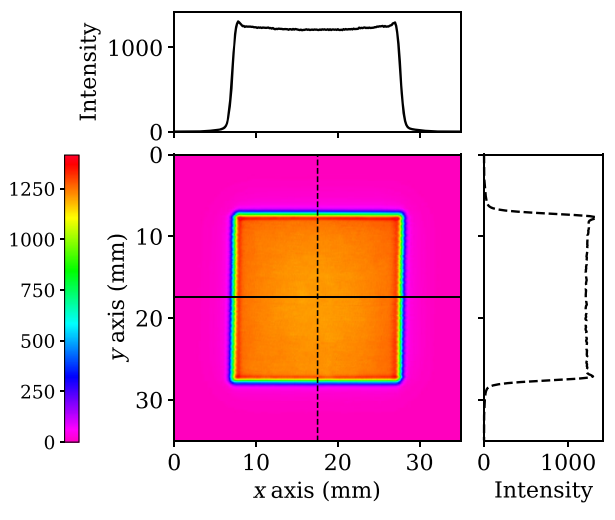

(a)

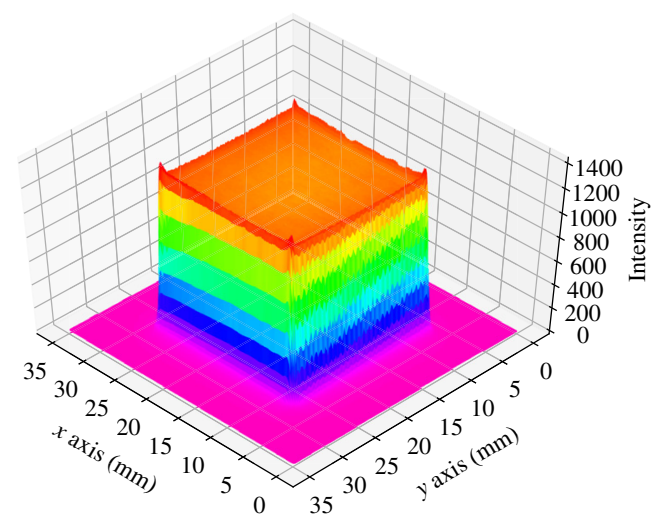

(b)

Fig. 10 Measured power density distribution of a 700-W, flat-top laser beam in the LAB process: (a) 2D contour plot with 1D slices along the $x$ axis (top) and $y$ axis (right) and (b) 3D contour plot. 
Table 1 ISO 13694:2018 and ISO 11145:2018 characterizing parameters of a 700-W, flat-top laser beam in the LAB system measured 10 consecutive times. Clip-level $\varepsilon=0.10$ and $\eta=0.80$.

\begin{tabular}{|c|c|c|c|}
\hline Characterizing parameter & Notation & Average & Standard deviation (\%) \\
\hline \multicolumn{4}{|l|}{ Beam power } \\
\hline Power & $P(z)$ & $28,576,928.9160 \mathrm{ADC}$ & 0.1796 \\
\hline Clip-level power & $P_{\eta}(z)$ & $26,175,447.0917$ ADC & 0.2915 \\
\hline \multicolumn{4}{|l|}{ Beam power density } \\
\hline Maximum power density & $E_{\max }(z)$ & $1,414.4310 \mathrm{ADC} / \mathrm{px}$ & 0.4033 \\
\hline Clip-level power density & $E_{\eta \mathrm{CL}}(z)$ & 1,131.5448 ADC/px & 0.4033 \\
\hline Clip-level average power density & $E_{\eta \text { ave }}(z)$ & $1,236.7589 \mathrm{ADC} / \mathrm{px}$ & 0.1895 \\
\hline \multicolumn{4}{|l|}{ Beam position } \\
\hline \multirow[t]{2}{*}{ Coordinate of maximum } & $x_{\max }$ & $128.5000 \mathrm{px}$ & 57.0124 \\
\hline & $y_{\max }$ & $199.9000 \mathrm{px}$ & 0.1582 \\
\hline \multirow[t]{2}{*}{ Beam centroid } & $\bar{x}(z)$ & $128.6000 \mathrm{px}$ & 0.4016 \\
\hline & $\bar{y}(z)$ & $127.0000 \mathrm{px}$ & 0.0000 \\
\hline \multirow[t]{2}{*}{ Beam positional stability } & $\Delta_{X}(z)$ & $2.0656 \mathrm{px}$ & - \\
\hline & $\Delta_{y}(z)$ & $0.0000 \mathrm{px}$ & - \\
\hline \multicolumn{4}{|l|}{ Effective beam size } \\
\hline \multirow[t]{2}{*}{ Beam width } & $d_{\sigma x}(z)$ & $176.7869 \mathrm{px}$ & 0.0555 \\
\hline & $d_{\sigma y}(z)$ & $177.7578 \mathrm{px}$ & 0.0685 \\
\hline \multirow[t]{2}{*}{ Clip-level irradiation area } & $A_{\varepsilon}^{i}(z)$ & $24,653.8000 \mathrm{px}$ & 0.2351 \\
\hline & $A_{\eta}^{i}(z)$ & $21,164.6000 \mathrm{px}$ & 0.3165 \\
\hline \multicolumn{4}{|l|}{ Beam shape } \\
\hline Beam aspect ratio & $\varepsilon(z)$ & 1.0055 & 0.0615 \\
\hline Fractional power & $f_{\eta}(z)$ & 0.9160 & 0.2823 \\
\hline Flatness factor & $F_{\eta}(z)$ & 0.8744 & 0.2464 \\
\hline Beam uniformity & $U_{\eta}(z)$ & 0.0219 & 1.7521 \\
\hline Plateau uniformity & $U_{p}(z)$ & 0.0284 & 8.0454 \\
\hline Edge steepness & $S_{\varepsilon, \eta}(z)$ & 0.1415 & 3.1772 \\
\hline
\end{tabular}

\subsubsection{Measurement unit of power density}

The measurement unit of power density can be converted from $\frac{\mathrm{ADC}}{\mathrm{px}}$ to $\frac{\mathrm{W}}{\mathrm{mm}^{2}}$ by combining the conversion factors of power and length. The conversion factor of power density is: $2.4495 \times 10^{-5} \frac{\mathrm{w}}{\mathrm{ADC}} \div\left(0.1371 \frac{\mathrm{mm}}{\mathrm{px}}\right)^{2}=0.0013 \frac{\mathrm{W}}{\mathrm{ADC}} \frac{\mathrm{px}^{2}}{\mathrm{~mm}^{2}}$.

\subsection{Characterizing Parameters}

\subsubsection{Beam power}

The power $P(z)$ has an average value of $700 \mathrm{~W}$ with a standard deviation of $0.1796 \%$, and the clip-level power $P_{\eta}(z)$ has an average value of $641.17 \mathrm{~W}$ with a standard deviation of $0.2915 \%$ considering a clip level of $\eta=0.80$. 


\subsubsection{Beam power density}

The maximum power density $E_{\max }(z)$ has an average value of $1.8387 \frac{\mathrm{W}}{\mathrm{mm}^{2}}$ with a standard deviation of $0.4033 \%$, the clip-level power density $E_{\eta \mathrm{CL}}(z)$ has an average value of $1.4710 \frac{\mathrm{w}}{\mathrm{mm}^{2}}$ with a standard deviation of $0.4033 \%$, and the clip-level average power density $E_{\eta \text { ave }}$ has an average value of $1.6079 \frac{\mathrm{W}}{\mathrm{mm}^{2}}$ with a standard deviation of $0.1895 \%$.

\subsubsection{Beam position}

The coordinate of maximum $\left(x_{\max }, y_{\max }, z\right)$ has an average value of $(17.6173 \mathrm{~mm}, 27.4063 \mathrm{~mm})$ with a standard deviation of $(57.0124 \%, 0.1582 \%)$. The high variation of the $x$ coordinate can be explained by looking at the coordinate of each individual measurement (Table 2). It is clear that one half of the measurements are centered at around $8.0889 \mathrm{~mm}$, whereas the other half are centered at around $27.1458 \mathrm{~mm}$. Considering each individual group, the new standard deviation is $0.0000 \%$ for the group at $8.0889 \mathrm{px}$ and $0.3571 \%$ for the group at $27.1458 \mathrm{~mm}$. The beam centroid $(\bar{x}(z), \bar{y}(z))$ is located at the coordinate $(17.6311 \mathrm{~mm}, 17.4117 \mathrm{~mm})$ with a beam positional stability $\left(\Delta_{x}(z), \Delta_{y}(z)\right)$ of $(0.2832 \mathrm{~mm}, 0.0000 \mathrm{~mm})$.

\subsubsection{Effective beam size}

The beam width $\left(d_{\sigma x}(z), d_{\sigma y}(z)\right)$ has an average value of $(24.2375 \mathrm{~mm}, 24.3706 \mathrm{~mm})$ with a standard deviation of $(0.0555 \%, 0.0685 \%)$ [Fig. 11(a)]. The clip-level irradiation areas $A_{\varepsilon}^{i}(z)$ and $A_{\eta}^{i}(z)$ have an average value of $463.4029 \mathrm{~mm}^{2}$ and $397.8185 \mathrm{~mm}^{2}$, respectively, with a corresponding standard deviation of $0.2351 \%$ and $0.3165 \%$.

One of the strong advantages of the LAB process is the selective heating capability, in which only the target device and its immediate surroundings are irradiated by the laser beam. In practical terms, this is achieved by setting the beam size to match the dimensions of the target device. However, the definition of beam size as described in Sec. 3.1.10 ${ }^{26}$ could pose a threat to the robustness of the LAB process since the edges of the target device could receive an insufficient amount of laser power, as discussed in Sec. 1. To get this point across, consider a die of size $13.00 \mathrm{~mm} \times 13.00 \mathrm{~mm}$ and a beam width of the same dimensions, as defined by Eqs. (9) and (10) [Fig. 11(b)]. It is evident that this arrangement has a high probability of yielding opencircuit issues at the peripheral solder bumps since the edges of the target device do not receive sufficient laser energy.

Table 2 Coordinate of maximum at location $z$ for each individual measurement.

\begin{tabular}{lcc}
\hline \hline Measurement & $x$ coordinate $(\mathrm{mm})$ & $y$ coordinate $(\mathrm{mm})$ \\
\hline 1 & 8.0889 & 27.4200 \\
2 & 8.0889 & 27.4200 \\
3 & 8.0889 & 27.4200 \\
4 & 8.0889 & 27.4200 \\
5 & 8.0889 & 27.2829 \\
6 & 27.0087 & 27.4200 \\
7 & 27.1458 & 27.4200 \\
8 & 27.1458 & 27.4200 \\
9 & 27.1458 & 27.4200 \\
10 & 27.2829 & 27.4200 \\
\hline \hline
\end{tabular}




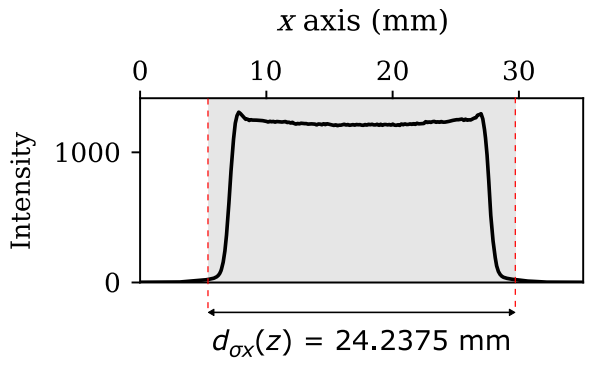

(a)

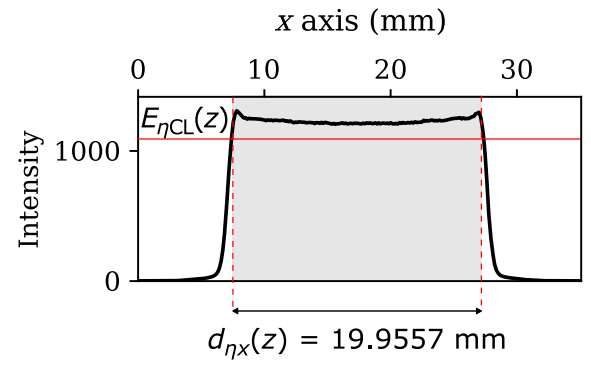

(c)

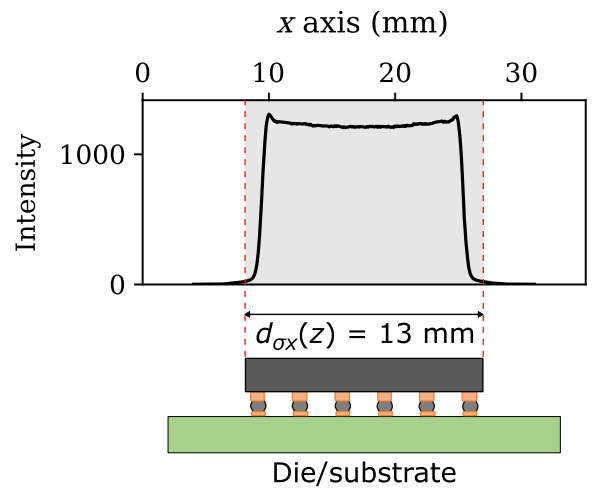

(b)

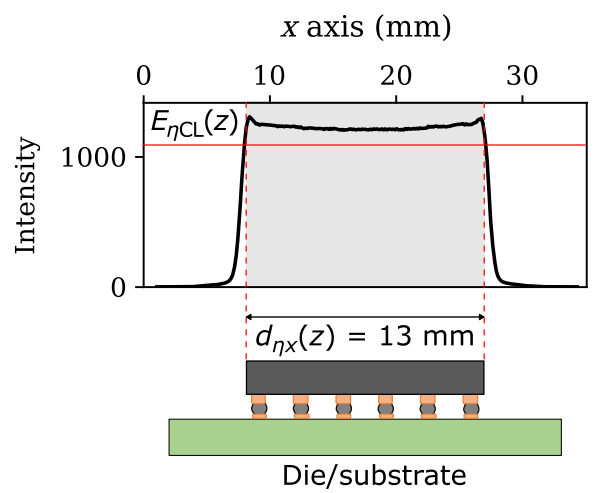

(d)

Fig. 11 Beam width definition and robustness of the LAB process. (a) The beam width $d_{\sigma x}(z)$ has an average value of $24.2375 \mathrm{~mm}$. (b) Example of a die of size $13.00 \mathrm{~mm} \times 13.00 \mathrm{~mm}$ and a beam width $d_{\sigma x}(z)$ of the same dimensions. The solder bumps at the periphery receive insufficient laser energy, which can lead to open-circuit issues. (c) The beam width $d_{\eta x}(z)$ has an average value of $19.9557 \mathrm{~mm}, 17.67 \%$ smaller than $d_{\sigma x}(z)$. (d) Example of a die of size $13.00 \mathrm{~mm} \times 13.00 \mathrm{~mm}$ and a beam width $d_{\eta x}(z)$ of the same dimensions. The solder bumps at the periphery receive sufficient laser energy, which reduces the risk of open-circuit issues.

At this point, the reader might realize that occasionally the ISO standards do not apply to their specific application, and that new definitions that suit their needs can be proposed. In this sense, we propose a new beam width definition that avoids this issue by combining the concepts of beam width and clip-level power density-the clip-level beam width $\left(d_{\eta x}(z), d_{\eta y}(z)\right)$. The clip-level beam width is calculated considering only contiguous pixels whose values are greater than $E_{\eta \mathrm{CL}}(z)$. Using this definition, the beam width has an average value of $(19.9557 \mathrm{~mm}$, $19.9288 \mathrm{~mm})$ with a standard deviation of $(0.3365 \%, 0.0552 \%)$ [Fig. 11(c)]. Once again, consider a die of size $13.00 \mathrm{~mm} \times 13.00 \mathrm{~mm}$ and a beam width of the same dimensions, but this time as defined by the clip-level beam width [Fig. 11(d)]. With this approach, one can be sure that the entire area of the target device receives sufficient laser energy and a robust solder interconnection is secured. Refer to Appendix A for the full definition of $\left(d_{\eta x}(z), d_{\eta y}(z)\right)$.

\subsubsection{Beam shape}

The beam aspect ratio $\varepsilon(z)$ has an average value of 1.0055, which is expected since the laser beam has a square shape. The fractional power $f_{\eta}(z)$ has an average value of 0.9160 , which indicates that $91.60 \%$ of the total power $P(z)$ has a value greater than the clip-level power $P_{\eta}(z)$. The flatness factor $F_{\eta}(z)$ has as average value of 0.8744 , only 12.56 percent points smaller than a perfectly flat-top power density distribution. The beam uniformity $U_{\eta}(z)$ has an average value of 0.0219 , which represents a variation of only $\pm 2.19 \% \mathrm{rms}$ from the 


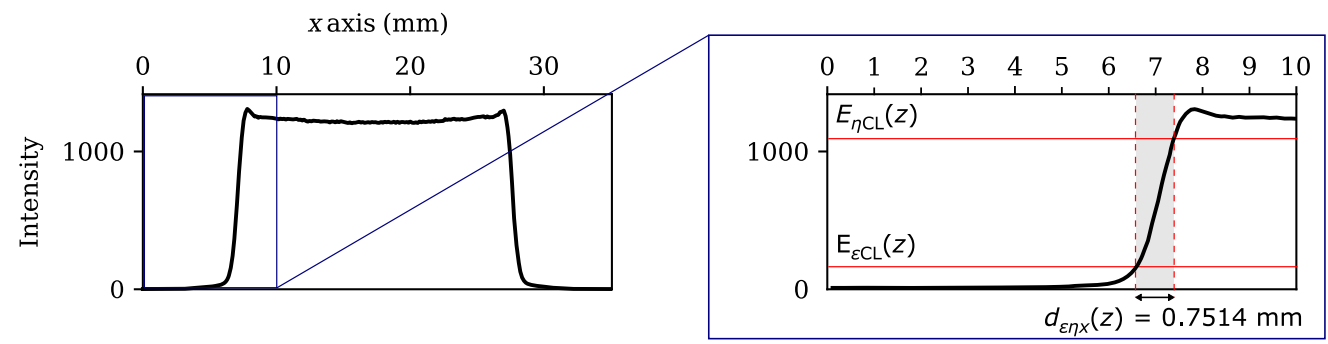

Fig. 12 Clip-level edge width definition. The edge width $d_{\varepsilon \eta \eta}(z)$ has an average value of $0.7514 \mathrm{~mm}$ with a standard deviation of $7.5791 \%$.

clip-level average power density $E_{\eta \text { ave }}(z)$. The plateau uniformity $U_{p}(z)$ has an average value of 0.0284 , which is just 2.84 percent points higher than a perfectly flat-top power density distribution. The edge steepness $S_{\varepsilon, \eta}(z)$ has an average value of 0.1415 , which indicates a power density distribution with fairly vertical edges.

During the LAB process, it is important to understand the influence that the laser emission onto a target device has on its neighboring components. This influence is expected to become more critical as the number of devices per substrate increases and the clearance between them decreases. Therefore, it is valuable to quantitatively determine a keep-out-zone outside of which the laser beam has negligible impact and devices are safe from undesired laser radiation. Because the edge steepness $S_{\varepsilon, \eta}(z)$ does not provide a straightforward explanation on this matter, we propose a new definition - the clip-level edge width. Here again, the reader might appreciate the possibility of proposing new definitions that better suit their specific application. The clip-level edge width $\left(d_{\varepsilon \eta x}(z), d_{\varepsilon \eta y}(z)\right)$ is calculated considering contiguous pixels whose values are in-between $E_{\varepsilon \mathrm{CL}}(z)$ and $E_{\eta \mathrm{CL}}(z)$ (Fig. 12). Using this definition, the edge width has an average value of $(0.7514 \mathrm{~mm}, 0.8556 \mathrm{~mm})$ with a standard deviation of $(7.5791 \%, 0.3823 \%)$. Refer to Appendix B for the full definition of $\left(d_{\varepsilon \eta x}(z), d_{\varepsilon \eta y}(z)\right)$.

\section{Conclusions}

In this paper, we have presented a didactic introduction to the theory of laser beam characterization and its application in the LAB process. As pointed out in Sec. 1, this paper should not be treated as a full review of the LAB process nor of the beam profiling instrument and measurement principle. These are only mentioned to enhance the reader's learning experience. Two ISO standards were studied in this paper, namely ISO 13694:2018 and ISO 11145:2018. Their characterizing parameters were presented, defined mathematically, and explained in detail through the use of an MWE. The reader was encouraged to follow the calculations step-by-step in order to verify the results.

The negative effects of background noise and digitizer baseline on the soundness of the beam analysis, in special calculations that involve weighted integral sums, were discussed. Three background noise correction methods were presented, one of which must be applied before carrying on the laser beam analysis. Furthermore, conversion between units of power (ADC and W), length (px and $\mathrm{mm}$ ), and power density (ADC/px and $\mathrm{W} / \mathrm{mm}^{2}$ ) were covered. It should be stressed that unit conversion is only valid when the output laser power is verified by an independent measurement tool, such as a power meter.

The average results of the 10 measurements showed that the laser system has an exceptionally consistent power density distribution, as demonstrated by the low standard deviation of nearly all characterizing parameters. Moreover, two new characterizing parameters that better suit the LAB process were proposed, namely the clip-level beam width and the cliplevel edge width. This proposal should be considered as an indication that researchers can, and oftentimes should, fully understand the ISO standards and propose new definitions that better suit their application, instead of simply relying on third-party, proprietary beam analysis software. 
Although this paper dealt with only two ISO standards, the methods learned from this paper can be easily applied to other norms that fit the reader's application. The Python source file containing the implementation of all discussed ISO definitions as well as the laser beam raw data can be made available upon reasonable request to the corresponding author.

\section{Appendix A: Proposal 1-Clip-Level Beam Width}

Clip-level beam width $\left(d_{\eta x}(z), d_{\eta y}(z)\right)$ is the irradiation width at location $z$ for which the power density $E\left(x_{p}, y_{p}, z\right)$ exceeds the clip-level power density $E_{\eta \mathrm{CL}}(z) .\left(d_{\eta x}(z), d_{\eta y}(z)\right)$ is mathematically expressed as

$$
\begin{aligned}
& d_{\eta x}(z)=\operatorname{COUNT}\left[E\left(x, y_{p}, z\right)>E_{\eta \mathrm{CL}}(z)\right], \\
& d_{\eta y}(z)=\operatorname{COUNT}\left[E\left(x_{p}, y, z\right)>E_{\eta \mathrm{CL}}(z)\right],
\end{aligned}
$$

where COUNT is a function that counts how many pixels have a value greater than the clip-level power density $E_{\eta \mathrm{CL}}(z)$. The clip-level beam width about the $x$ axis is computed by iterating along the row indices [Fig. 13(a)] and the clip-level beam width about the $y$ axis is computed by iterating along the column indices [Fig. 13(b)]. The results for each axis are then averaged out disregarding the zeros and any eventual outliers. The use of a high-pixel resolution is recommended in order to avoid quantization errors that may arise from counting pixels. The clip-level beam width of the MWE (Fig. 5) is $d_{\eta x}(z)=3$ and $d_{\eta y}(z)=3$ considering a clip-level of $\eta=0.80$.

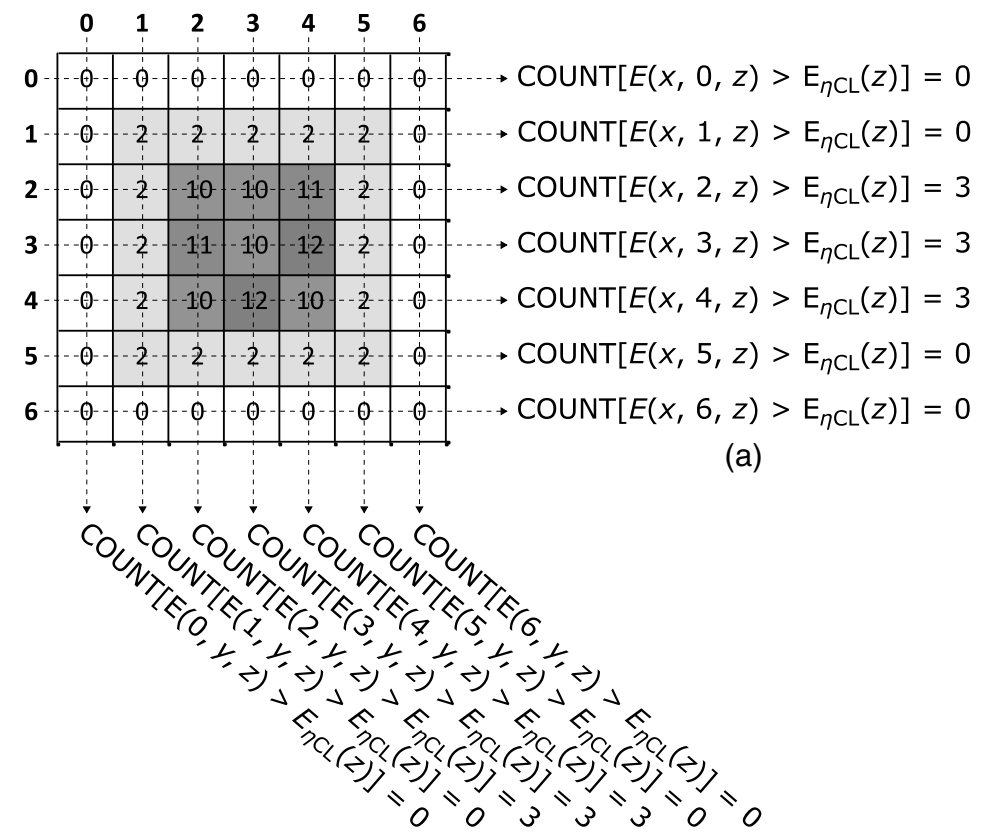

(b)

Fig. 13 Visual explanation of the clip-level beam width. (a) The clip-level beam width about the $x$ axis is computed by iterating along the row indices. The nonzero values are then averaged out disregarding any eventual outliers. (b) The clip-level beam width along the $y$ axis is computed by iterating along the column indices. The nonzero values are then averaged out disregarding any eventual outliers. 


\section{Appendix B: Proposal 2-Clip-Level Edge Width}

Clip-level edge width $\left(d_{\varepsilon \eta x}(z), d_{\varepsilon \eta y}(z)\right)$ is the irradiation width at location $z$ for which the power density $E\left(x_{p}, y_{p}, z\right)$ is enclosed by the power densities $E_{\varepsilon \mathrm{CL}}(z)$ and $E_{\eta \mathrm{CL}}(z) \cdot\left(d_{\varepsilon \eta x}(z), d_{\varepsilon \eta y}(z)\right)$ is mathematically defined as

$$
\begin{aligned}
& d_{\varepsilon \eta x}(z)=\frac{\operatorname{COUNT}\left[E_{\varepsilon \mathrm{CL}}(z)<E\left(x, y_{p}, z\right)<E_{\eta \mathrm{CL}}(z)\right]}{2}, \\
& d_{\varepsilon \eta y}(z)=\frac{\operatorname{COUNT}\left[E_{\varepsilon \mathrm{CL}}(z)<E\left(x_{p}, y, z\right)<E_{\eta \mathrm{CL}}(z)\right]}{2},
\end{aligned}
$$

where COUNT is a function that counts how many pixels have a value in-between the clip-level power densities $E_{\varepsilon \mathrm{CL}}(z)$ and $E_{\eta \mathrm{CL}}(z)$. The clip-level edge width about the $x$ axis is computed by iterating along the row indices [Fig. 14(a)] and the clip-level edge width about the $y$ axis is computed by iterating along the column indices [Fig. 14(b)]. The results for each axis are averaged out disregarding the zeros and any eventual outliers, and the resulting value is then divided by two in consideration of the two opposite edges. The use of a high-pixel resolution is recommended in order to avoid quantization errors that may arise from counting pixels. The clip-level edge width of the MWE (Fig. 5) is $d_{\varepsilon \eta x}(z)=1$ and $d_{\varepsilon \eta y}(z)=1$ considering a clip level of $\varepsilon=0.10$ and $\eta=0.80$.

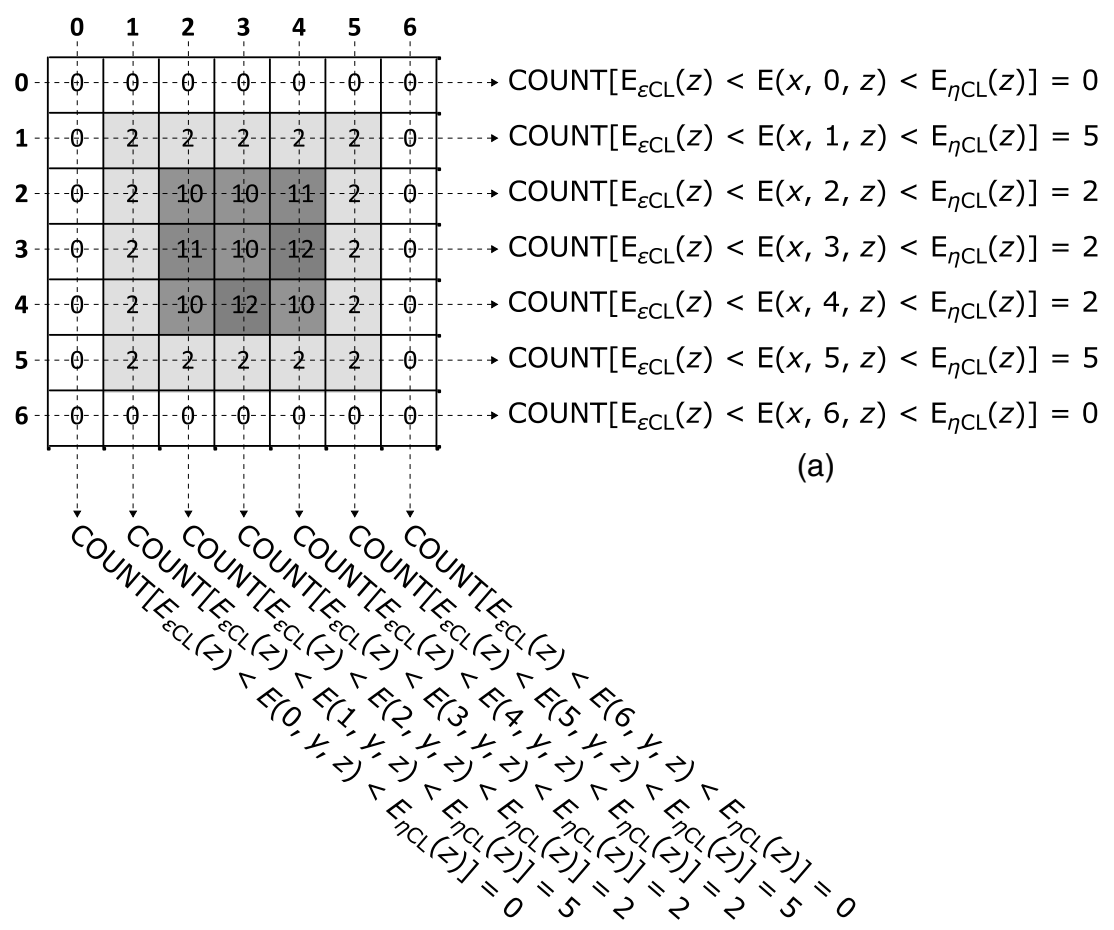

(b)

Fig. 14 Visual explanation of the clip-level edge width. (a) The clip-level edge width about the $x$ axis is computed by iterating along the row indices. The nonzero values are averaged out disregarding any eventual outliers, and the resulting value is divided by two. (b) The clip-level beam edge along the $y$ axis is computed by iterating along the column indices. The nonzero values are averaged out disregarding any eventual outliers, and the resulting value is divided by two. 


\section{Acknowledgments}

The authors would like to extend their sincere gratitude to JCET Korea R\&D Division, who shared valuable insights for the better development of this project.

\section{References}

1. V. Krutikov et al., "System of ISO standards for characterization of optical components and laser beams," Proc. SPIE 4900, 339-341 (2002).

2. K. Gindele, "Standardization of characteristics of optical components and laser beams," Proc. SPIE 2870, 2-10 (1996).

3. W. Kluft, "Measuring principles and laser beam characterization procedures," Proc. SPIE 2870, 340-352 (1996).

4. R. Maestle et al., "Instruments and standard measurement procedures for laser beam characterization," Proc. SPIE 3405, 978-989 (1998).

5. K. R. Mann, J. Ohlenbusch, and V. Westphal, "Characterization of excimer laser beam parameters," Proc. SPIE 2870, 367-377 (1996).

6. C. Wild, "Examination of standards and test procedures for laser beam characterization of $\mathrm{CO}_{2}$ industrial lasers," Proc. SPIE 2870, 312-318 (1996).

7. P. Apte and M. C. Gower, "Practical implementation of test methods for the characterization of laser beam power and energy density distribution," Proc. SPIE 2870, 40-49 (1996).

8. K. Mann et al., "Comprehensive laser beam characterization for applications in material processing," Proc. SPIE 7202, 72020C (2009).

9. D. G. Sporea and M. V. Udrea, "Beam characterization of commercial red and infrared diode lasers," Proc. SPIE 5227, 227-232 (2003).

10. D. G. Sporea et al., "Laser beam characterization under PC control," Proc. SPIE 4430, 852-857 (2001).

11. P. Di Lazzaro et al., "Flat-top shaped laser beams: reliability of standard parameters," Proc. SPIE 5777, 705-710 (2005).

12. S. Bollanti et al., "Edge steepness and plateau uniformity of a nearly flat-top-shaped laser beam," Appl. Phys. B 78(2), 195-198 (2004).

13. D. G. Sporea, G. Dumitru, and B. Prelipcean, "Computation algorithms for laser beam visualization," Proc. SPIE 3736, 328-335 (1999).

14. T. Visan and D. Sporea, "PC control and data processing in relation with a standalone laser beam analyzer," Rev. Sci. Instrum. 71(6), 2610-2611 (2000).

15. Y. Jung et al., "Development of next generation flip chip interconnection technology using homogenized laser-assisted bonding," in IEEE 66th Electron. Comp. and Technol. Conf., IEEE, pp. 88-94 (2016).

16. C. Kim et al., "Development of extremely thin profile flip chip CSP using laser assisted bonding technology," in IEEE CPMT Symp. Jpn., IEEE, pp. 45-49 (2017).

17. K.-S. Choi et al., "Development of stacking process for 3D TSV (through silicon via) structure using laser," Int. Symp. Microelectron. 2017(1), 000067 (2017).

18. W. A. Braganca et al., "Collective laser-assisted bonding process for 3D TSV integration with NCP," ETRI J. 41(3), 396-407 (2019).

19. K.-S. Choi et al., "Interconnection process using laser and hybrid underfill for LED array module on pet substrate," in IEEE 68th Electron. Comp. and Technol. Conf., IEEE, pp. 1567-1573 (2018).

20. W. A. Braganca, K. K. Oe, and K. Y. Cheol, "Development of a laser-assisted bonding process for a flip-chip die with backside metallization," in IEEE 22nd Electron. Packaging Technol. Conf., IEEE, pp. 68-72 (2020).

21. F. M. Dickey, Laser Beam Shaping: Theory and Techniques, CRC Press (2018).

22. R. Voelkel and K. J. Weible, "Laser beam homogenizing: limitations and constraints," Proc. SPIE 7102, 71020J (2008).

23. J. A. Hoffnagle and C. M. Jefferson, "Design and performance of a refractive optical system that converts a Gaussian to a flattop beam," Appl. Opt. 39(30), 5488-5499 (2000). 
24. PRIMES, “BeamMonitor BM+ with LDS 2.98,” 01/2019 en ed., 2019, https://www.primes .de/en/products/beam-distribution/raw-beam-measurement/beammonitor-bmplus.html

25. "Optics and photonics-lasers and laser-related equipment - test methods for laser beam power (energy) density distribution," ISO 13694:2018, International Organization for Standardization (2018).

26. "Optics and photonics—-lasers and laser-related equipment—vocabulary and symbols," ISO 11145:2018, International Organization for Standardization (2018).

Wagno Alves Braganca received his BEng degree in electrical engineering with an emphasis in microelectronics from the Federal University of Minas Gerais, Belo Horizonte, Brazil, in 2016 and his MSEng degree in advanced device technology from the University of Science and Technology, Daejeon, Republic of Korea, in 2018. He worked as a research assistant at the Electronics and Telecommunications Research Institute, Daejeon, Republic of Korea, from 2016 to 2018, where he researched and developed through-silicon via integration processes. Currently, he is an R\&D engineer at JCET Korea, where he works with LAB process.

KyungOe Kim received his bachelor's degree in material science and engineering from Hanyang University, Seoul, Republic of Korea, in 1996 and his master's degree from the Surface Engineering Laboratory, Hanyang University, Seoul, Republic of Korea, in 1999. After joining Chippac Korea in 2000, he has worked as a research engineer, especially for the development of FlipChip technology from C4 bumped FC to $\mathrm{Cu}$ bumped FC. Now, he is an $\mathrm{R} \& \mathrm{D}$ director at JCET Korea, responsible for new interconnect technology development. 